A Study on the Growth Policies and Profitability of Select Mergers and Acquisitions in India

\author{
A. R. Sindhu \\ S. Madhavan \\ AMET International Journal of Management \\ ISSN 2231-6779 \\ July - Dec 2016 \\ Vol. 10, pp. $42-68$
}




\title{
A STUDY ON THE GROWTH POLICIES AND PROFITABILITY'S OF SELECT MERGERS AND ACQUISITIONS IN INDIA
}

\author{
A R Sindhu \\ Dr. S. Madhavan
}

\section{Abstract}

The study aims to investigate the growth percentage in Earning per Share, Net worth, Debt equity Ratio and Market value of companies starting from Jan 2014 to March 2014, which is carried out on a sample of 22 companies, who under vent M\&A. It is assumed that Merger and Acquisition (M\&A) develop the company financial performance, organisational competitiveness and it is an easier way to internationalisation also. 2014 was a year for very strong foreign investor interest in India, while the economic and growth sentiments are enormously positive. The present research paper was based on Secondary data from the CMIE's Prowess database and Annual Reports of the companies concerned. Finally from the results it is concluded that mergers have a significant change in enhancing the profitability of acquirer companies.

Keywords: Mergers and Acquisitions, Earning Per Share, Debt Equity Ratio, Networth and Market value of company

\section{Introduction}

Corporate Restructuring is the procedure required in changing the association of a business. Corporate rebuilding can include rolling out emotional improvements to a business by removing or consolidating divisions that frequently has the impact of dislodging staff individuals. In today's globalized economy, Mergers and Acquisitions are by and large progressively utilized as a target for speedier development in piece of the pie, accomplishing a bigger size, to improve gainfulness, resource base, and broadening of hazard and for turning out to be more focused through economies of scale.

Each merger has its own particular novel reasons as the consolidating of two organizations is a decent business choice. As the term Merger implies converging of two organizations where one new organization will keep on existing. Reverse mergers (also known as a reverse takeover or reverse IPO) are a very popular in Merger. A reverse merger is a way for private companies to go public, typically 
through a simpler, shorter, and less expensive process. Mergers can be categorized as follows Horizontal Merger, Vertical Merger and Conglomerate Merger. The Merger and Acquisition Process can be separated into five stages, they are:

Table 1: Merger \& Acquisition Phases

\begin{tabular}{|l|l|}
\hline \multicolumn{1}{|c|}{ Phases } & \multicolumn{1}{c|}{ Process } \\
\hline Phase 1 & Pre Acquisition Review \\
\hline Phase 2 & Search \& Screen Targets \\
\hline Phase 3 & Investigate \& Value the Target \\
\hline Phase 4 & Acquire through Negotiation \\
\hline Phase 5 & Post Merger Integration. \\
\hline
\end{tabular}

\section{An Overview of M\&A in 2014}

As indicated by the information discharged by Grant Thornton India, an Accounting and Consulting firm, as the aggregate Merger and Acquisitions (M\&A) bargains amid the year 2014 were esteemed at US $\$ 38$ billion (573 arrangements) when contrasted with US $\$ 28.19$ billion (500 arrangements) amid the comparing time frame in 2013.

As 2013 M\&A demonstrated an exceptionally baffling business sector, the raise of 2014 was astounding with a full speed of Mega arrangements in the main quarter itself. M\&A movement in 2014 relies on upon various elements, which incorporates Economic Conditions, Private Equity Impact, Venture Capital Pipeline and Intellectual Property Motivations. The main 5 M\&A segments in the year 2014 were distinguished as Pharmaceuticals, Healthcare and Biotechnology, Telecommunication, Transport and Logistics, Retail and Consumer and Energy and Natural Resources. 
Table 2: 2014 Overall M\&A

\begin{tabular}{|c|c|c|c|c|c|c|}
\hline & \multirow{3}{*}{ Year } & \multicolumn{4}{|c|}{ Deal Summary } & \multirow{3}{*}{ Total } \\
\hline & & \multirow{2}{*}{ Domestic } & \multicolumn{2}{|c|}{ Cross-border } & \multirow{2}{*}{$\begin{array}{l}\text { Merger and } \\
\text { Internal } \\
\text { Restructuring }\end{array}$} & \\
\hline & & & Inbound & Outbound & & \\
\hline \multirow{2}{*}{ 竞 } & $\begin{array}{c}\text { Value } \\
\text { (US\$ } \\
\text { mn) }\end{array}$ & 16,316 & 11,829 & 5,981 & 3,989 & 38,115 \\
\hline & Volume & 253 & 166 & 117 & 37 & 573 \\
\hline \multirow{2}{*}{$\stackrel{m}{\stackrel{m}{\circ}}$} & $\begin{array}{l}\text { Value } \\
\text { (US\$ } \\
\text { mn) }\end{array}$ & 5,636 & 8,736 & 9,249 & 4,541 & 28,162 \\
\hline & Volume & 217 & 139 & 82 & 58 & 497 \\
\hline
\end{tabular}

Source : Grand Thornton Deal tracker 2014

\section{Why Companies Acquire?}

The Indian market is substantial, assorted and complex and for global organizations assessing a passage, it is basic to have a home-developed admonitory group that comprehends the market, appreciates close associations with chiefs in organizations, can oversee singular promoter sensitivities, and in addition comprehends the subtleties of Indian controls. Such an affiliation would help organizations explore the social, political, administrative and legitimate systems easily.

While India is among the most remunerating spots to work together in, it accompanies its own arrangement of interesting difficulties. There are a few experiences from the point of view of an inbound acquirer and practices why an organization mostly go for a get, a portion of the real reasons are because of 
enhanced market get to, consolidated business creation, co-ordinated Strategies, shared know-how, vertical incorporation and shared unmistakable assets.

Table 3: Deal activity by industry

\begin{tabular}{|l|r|r|r|r|}
\hline \multirow{2}{*}{ Target vertical } & \multicolumn{2}{|c|}{$\mathbf{2 0 1 3}$} & \multicolumn{2}{c|}{$\mathbf{2 0 1 4}$} \\
\cline { 2 - 5 } & velume & \multicolumn{1}{c|}{$\begin{array}{c}\text { Deal value } \\
\text { (US\$ } \\
\text { million) }\end{array}$} & $\begin{array}{c}\text { Deal } \\
\text { volume }\end{array}$ & $\begin{array}{r}\text { Deal } \\
\text { value } \\
\text { (US\$ } \\
\text { million) }\end{array}$ \\
\hline Cement and building products & 16 & 992 & 20 & 1,147 \\
\hline Chemicals & 30 & 415 & 30 & 290 \\
\hline Diversified Industrial Products & 79 & 399 & 78 & 1,244 \\
\hline Financial services & 56 & 1,342 & 55 & 3,751 \\
\hline Health care & 26 & 663 & 34 & 295 \\
\hline Pharmaceuticals & 32 & 3,717 & 52 & 4,159 \\
\hline Metals and mining & 35 & 446 & 26 & 676 \\
\hline Media and entertainment & 51 & 83 & 53 & 1,094 \\
\hline Infrastructure & 73 & 1,983 & 94 & 5,829 \\
\hline Professional services & 29 & 487 & 45 & 108 \\
\hline Real estate & 24 & 639 & 32 & 1,331 \\
\hline Retail and consumer products & 73 & 3,843 & 96 & 4,010 \\
\hline Technology & 71 & 544 & 116 & 2,021 \\
\hline
\end{tabular}

\section{Source: EV analysis of Thomson ONE data}

\section{Reasons for M \& A:}

One very important reason for M\&A is Synergy value element. The joining or merging of the two companies creates an additional value called Synergy Value, to make it simple $2+2=5$. Synergy value can take three forms Revenues, Expenses, Cost of Capital.

If the expected synergy values may not be realized, the merger is considered to be a failure. Some of the reasons behind failed mergers are Poor 
strategic fit, Cultural and Social Differences Incomplete and Inadequate Due Diligence, Poorly Managed Integration, Paying too Much, Overly Optimistic.

\section{Brief Literature Survey:}

\section{Table 4: Studies on M\&A in India - A Brief Literature Survey}

\begin{tabular}{|c|c|c|c|}
\hline Title & Study by & Period & Objectives/Tools/Findings \\
\hline $\begin{array}{l}\text { 1.Impact of } \\
\text { Mergers on } \\
\text { Corporate } \\
\text { Performance in } \\
\text { India }\end{array}$ & $\begin{array}{c}\text { Amarjit Saini \& } \\
\text { Dr.Ravi Singla }\end{array}$ & 2015 & $\begin{array}{l}\text { Obj: To analyze the impact of } M \& A \\
\text { on the financial performance of } \\
\text { acquirer companies and to examine } \\
\text { the instantaneous impact of } M \& A \\
\text { on acquirer companies } \\
\text { Shareholders wealth. } \\
\text { Tools: Ratios, T-test, Z-test, Event } \\
\text { Window } \\
\text { Fin: The acquirer companies have } \\
\text { not observed any significant impact } \\
\text { on their Liquidity and Profitability } \\
\text { Position. }\end{array}$ \\
\hline $\begin{array}{l}\text { 2.Impact of } \\
\text { Mergers \& } \\
\text { Acquisitions on } \\
\text { Firms' Long } \\
\text { Term } \\
\text { Performance: A } \\
\text { Pre \& Post } \\
\text { Analysis of the } \\
\text { Indian Telecom } \\
\text { Industry }\end{array}$ & $\begin{array}{c}\text { Neha Verma, } \\
\text { Dr. Rahul Sharma }\end{array}$ & 2014 & $\begin{array}{l}\text { Obj: To analyze the impact and to } \\
\text { identify M\&A induced changes of } \\
\text { select financial and operating } \\
\text { performance variables on Return on } \\
\text { Shareholder's Fund (ROSF) of the } \\
\text { companies and synergies, if any, } \\
\text { resulting from M\&A's in the Indian } \\
\text { Telecom Sector during the period } \\
\text { 2001- } 02 \text { to 2007-08. } \\
\text { Tools: Financial \& Operating } \\
\text { Performance Variables, t-test } \\
\text { Fin: Managers must retain focus on } \\
\text { firm's business, particularly, post- } \\
\text { M\&A integration process to achieve } \\
\text { the real } \\
\text { objectives of the M\&A. }\end{array}$ \\
\hline & & & Obj: To analyse the Trends of $M \& A$ \\
\hline
\end{tabular}




\begin{tabular}{|c|c|c|c|}
\hline $\begin{array}{l}\text { 3.Mergers \& } \\
\text { Acquisitions in } \\
\text { India: } \\
\text { A sectoral } \\
\text { analysis }\end{array}$ & Priya Bhalla & 2014 & $\begin{array}{l}\text { in various Sectors with special } \\
\text { focus on Indian Financial Sector } \\
\text { and to investigate the differential } \\
\text { representation of various sectors in } \\
\text { M\&A. } \\
\text { Fin: From the study it is observed } \\
\text { that Pharmaceutical, Telecom and } \\
\text { Financial Sectors have witnessed } \\
\text { most fundamental reforms and } \\
\text { India is lagging behind other } \\
\text { advanced and emerging economies } \\
\text { in terms of number and value of } \\
\text { M\&A. }\end{array}$ \\
\hline $\begin{array}{l}\text { 4.Impact of M\&A } \\
\text { on Operating } \\
\text { Performance: } \\
\text { Evidence from } \\
\text { Manufacturing } \\
\text { Firms in India }\end{array}$ & $\begin{array}{c}\text { Ramachandran } \\
\text { Azhagaiah \& } \\
\text { Thangavelu } \\
\text { Sathishkumar }\end{array}$ & 2014 & $\begin{array}{l}\text { Obj: To analyse the effect of M\&A } \\
\text { on the attributes of Operating } \\
\text { Performance Vs Gross Earnings, } \\
\text { Liquidity, Financial Risk, Cost of } \\
\text { Utilisation, Turnover, Growth and } \\
\text { Operating Leverage and to study } \\
\text { shift-in-structure in the Operating } \\
\text { Performance for post merger period } \\
\text { of acquiring manufacturing firms of } \\
\text { India } \\
\text { Tool: Factor Analysis, Correlation } \\
\text { Matrix, Multiple Regression Analysis } \\
\text { and Chow test } \\
\text { Fin: The M\&A process has } \\
\text { significant effect on OP of acquiring } \\
\text { Manufacturing firms of India } \\
\text { After M\&A. }\end{array}$ \\
\hline $\begin{array}{l}\text { 5.M\&A: An } \\
\text { Empirical study } \\
\text { on the Post } \\
\text { Merger } \\
\text { performance of } \\
\text { selected } \\
\text { Corporate Firms }\end{array}$ & Rosy Kalra & 2013 & $\begin{array}{l}\text { Obj: To study the impact of M\&A } \\
\text { on the Operating Performance, } \\
\text { Liquidity, Profitability and Leverage } \\
\text { of merged/acquirer by considering } \\
3 \text { years (April 08-March 09) on } \\
\text { evaluating Pre and Post merger } \\
\text { process of selected companies. } \\
\text { Tool: Financial Ratio, T-test } \\
\text { Fin: The result of the study shows }\end{array}$ \\
\hline
\end{tabular}




\begin{tabular}{|c|c|c|c|}
\hline in India & & & $\begin{array}{l}\text { that } M \& A \text { have a significant impact } \\
\text { on the } L, P \text {, and } O P \text {, moreover the } \\
\text { management cannot take it for } \\
\text { granted that synergy will be } \\
\text { generated and profits will increase } \\
\text { simply by going for } M \& A \text {. }\end{array}$ \\
\hline $\begin{array}{l}\text { 6.M\&A: A growth } \\
\text { perspective from } \\
\text { India }\end{array}$ & $\begin{array}{c}\text { Prof. Babasaheb. R. } \\
\text { Jadhav , } \\
\text { Dr.D.B.Bharati }\end{array}$ & 2013 & $\begin{array}{l}\text { Obj: To examine the strategies of } \\
\text { M\&A in India from growth } \\
\text { prospective and cause \& effect } \\
\text { analysis and to study the FDI in } \\
\text { M\&A deals. } \\
\text { Tool: Line chart, Bar Diagram, } \\
\text { Percentage and Proportions. } \\
\text { Fin: FDI in acquisition is double } \\
\text { than the merger and there are lot } \\
\text { of disparities between deals and } \\
\text { values of acquisition is more in total } \\
\text { M\&A. }\end{array}$ \\
\hline $\begin{array}{l}\text { 7.Determinants } \\
\text { of M\&A of firms } \\
\text { in the Indian } \\
\text { Financial Sector: } \\
\text { An Empirical } \\
\text { Analysis }\end{array}$ & Priya Bhalla & 2011 & $\begin{array}{l}\text { Obj: To investigate the motives of } \\
288 \text { entities belonging to Financial } \\
\text { Services Sector of India for the } \\
\text { period } 1997-98 \text { to } 2007-08 \\
\text { Tool: CAMEL rating system, } \\
\text { Correlation, Logit-probit analysis. } \\
\text { Fin: It supports the 'market for } \\
\text { control' hypothesis and one of the } \\
\text { motives for acquiring other firms in } \\
\text { the Financial Sector may be to } \\
\text { obtain greater Liquidity and } \\
\text { Diversification in Operations. }\end{array}$ \\
\hline $\begin{array}{l}\text { 8.M\&A - "A } \\
\text { change paradigm } \\
\text { in performance } \\
\text { of Indian } \\
\text { company }\end{array}$ & $\begin{array}{l}\text { Prof. Natika Jain \& } \\
\text { Prof.Sulbha Raorane }\end{array}$ & 2011 & $\begin{array}{l}\text { Obj: To analysis the impact of } M \& A \\
\text { on the Performance of the selected } \\
\text { companies in Indian context. } \\
\text { Tool: Ratio analysis \& T-test } \\
\text { Fin: The acquirer companies } \\
\text { always benefited more than the } \\
\text { targeted companies in the merger } \\
\text { event. }\end{array}$ \\
\hline
\end{tabular}




\begin{tabular}{|c|c|c|c|}
\hline $\begin{array}{l}\text { 9.Mergers in } \\
\text { Indian Industry: } \\
\text { performance } \\
\text { and impacting } \\
\text { factors. }\end{array}$ & K. Ramakrishnan & 2010 & $\begin{array}{l}\text { Obj: Long-term post-merger firm } \\
\text { performance measurement. To } \\
\text { analyses of the impact of the } \\
\text { strategic factors on long-term post- } \\
\text { merger firm performance. } \\
\text { Tool: cross-sectional linear } \\
\text { regression model, paired-samples t- } \\
\text { test } \\
\text { Fin: Unrelated mergers appear to } \\
\text { be performing better in the long } \\
\text { term, contrary to strategic } \\
\text { management theory. }\end{array}$ \\
\hline $\begin{array}{l}\text { 10.Type of } \\
\text { Merger and } \\
\text { Impact on } \\
\text { Operating } \\
\text { Performance: } \\
\text { The Indian } \\
\text { Experience }\end{array}$ & $\begin{array}{l}\text { Pramod Mantravadi \& } \\
\text { A Vidyadhar Reddy }\end{array}$ & 2008 & $\begin{array}{l}\text { Obj: To analyse Post-merger } \\
\text { Operating Performance for } \\
\text { acquiring firms in India which are } \\
\text { involved in different types of } \\
\text { mergers. } \\
\text { Tool: Ratio Analysis } \\
\text { Fin: It is suggested that Horizontal } \\
\text { merger had caused the highest } \\
\text { decline in the OP of merging } \\
\text { companies. }\end{array}$ \\
\hline $\begin{array}{l}\text { 11. Relative size } \\
\text { in Mergers and } \\
\text { Operating } \\
\text { Performance: } \\
\text { Indian } \\
\text { Experience }\end{array}$ & $\begin{array}{l}\text { Pramod Mantravadi \& } \\
\text { A Vidyadhar Reddy }\end{array}$ & 2007 & $\begin{array}{l}\text { Obj: Analysing the Post-merger } \\
\text { Operating Performance for } \\
\text { acquiring firms in Industry during } \\
\text { Post-reform period. } \\
\text { Tool: Ratio Analysis, Paired - t-test } \\
\text { Fin: The analysis of Pre and Post } \\
\text { merger OP ratios for the acquiring } \\
\text { firms in the sample seems to } \\
\text { indicate the relative size does make } \\
\text { some difference to the Post-merger } \\
\text { OP of acquiring firms. }\end{array}$ \\
\hline $\begin{array}{l}\text { 12.Post-merger } \\
\text { corporate } \\
\text { performance: an } \\
\text { Indian } \\
\text { perspective }\end{array}$ & Raj Kumar & 2009 & $\begin{array}{l}\text { Obj: To measure pre-merger size- } \\
\text { and industry-adjusted performance } \\
\text { of the acquiring and acquired } \\
\text { companies, combined into one } \\
\text { measure and post merger } \\
\text { operating performance of the }\end{array}$ \\
\hline
\end{tabular}




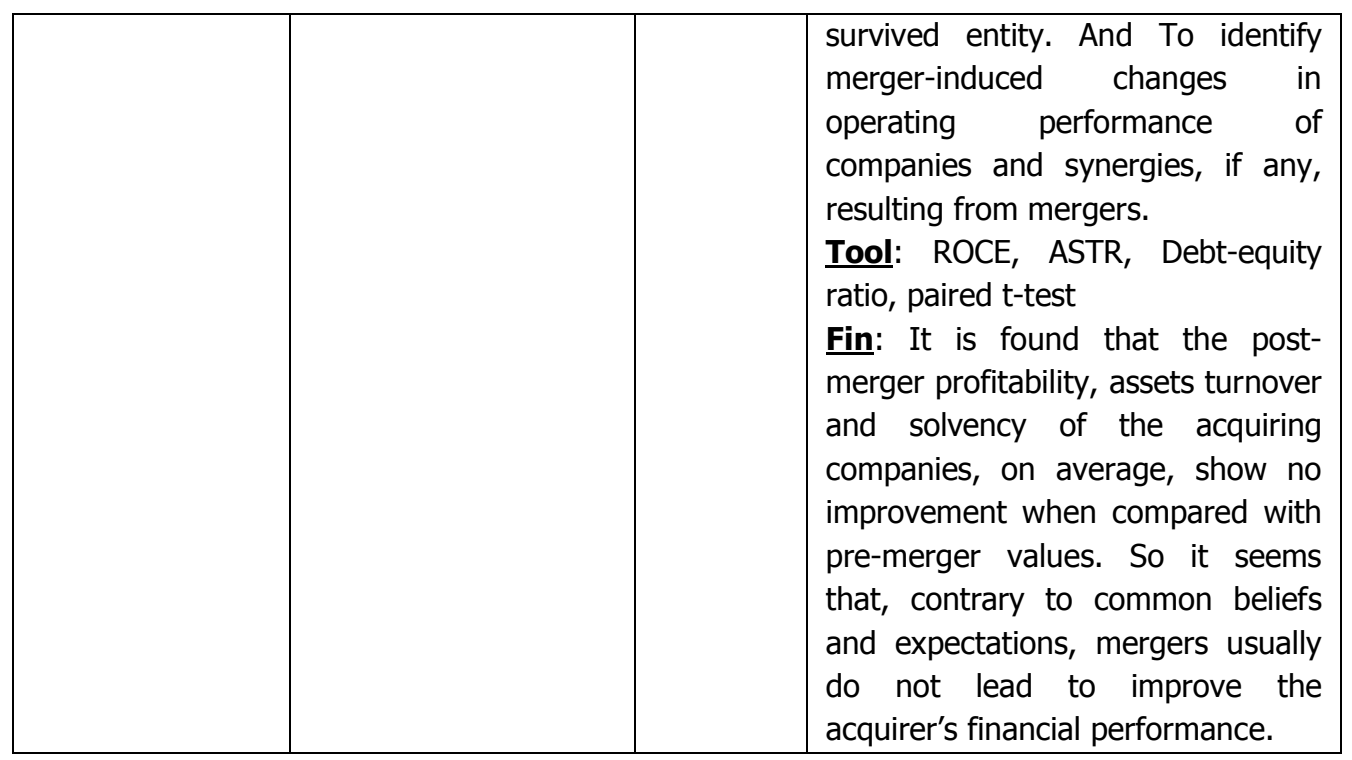

\section{Major findings from reviews:}

The common finding that can be drawn from the above research studies was that various analysis were made in the area of operating performance of acquiring firms in different industries only. As per the analysis made in operating performance, it was noted that there was a significant effect on acquiring firms while going for M\&A. There were only few studies made under sector wise and the types of mergers. The main important point of the acquirer companies was that the targeted companies were always benefited more in the M\&A event.

\section{Research Gap}

Most of the studies were based on the yearly data focusing on the impact of Operating Performance of acquiring firms using various attributes such as Liquidity, Profitability and Leverage. However, very little number of study were made on the growth attribute showing whether there was a decline or growth in Pre and Post M\&A from the above reviews. So the researcher has focused on calculating the growth percentage on Pre and Post M\&A. 


\section{Research Methodology:}

\section{Objectives}

- To analyse the growth percentage of M\&As on various attributes like Earnings Per Share, Net Worth, Debt Equity Ratio and Market Value of Company to consider Investment Opportunities.

\section{Data collection and Methodology}

Descriptive research design is used and Convenience Sampling has been employed to select the sample companies. The data were collected from 2014 period announced Merged and Acquired companies during 3 months (January, February and March 2014) in which a sample of 22 selected companies are taken for the study.

The announced Merged and Acquired companies were taken from the Centre for Monitoring Indian Economy (CMIE) Prowess database. The study was mainly based on secondary data, which were also from companies Annual Reports, UNCTAD cross-border M\&A database 2013 \& 2014, BSE website, Grand Thornton Dealtracker 2014 and from various other reports, journals and books.

\section{Analysis and Interpretation}

5.Table - Calculation on Growth percentage on Earnings Per Share of M\&A firms:

\begin{tabular}{|c|c|c|c|c|c|c|c|c|c|}
\hline \multirow[b]{2}{*}{$\begin{array}{l}\frac{0}{2} \\
\dot{\omega}\end{array}$} & \multirow[b]{2}{*}{ 高 } & \multirow[b]{2}{*}{ 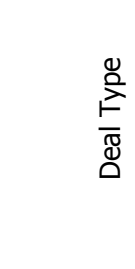 } & \multirow[b]{2}{*}{ 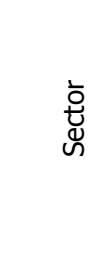 } & \multirow{2}{*}{ 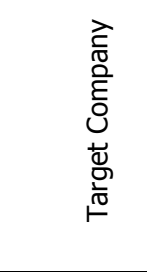 } & \multirow[b]{2}{*}{ 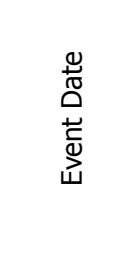 } & \multicolumn{4}{|c|}{ Earnings Per Share } \\
\hline & & & & & & 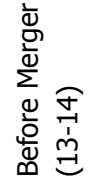 & 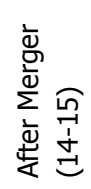 & 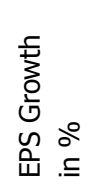 & 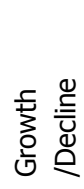 \\
\hline \multicolumn{10}{|c|}{ January } \\
\hline 1 & $\begin{array}{l}\text { Sunteck } \\
\text { Realty Ltd. }\end{array}$ & Merger & $\begin{array}{l}\text { Real } \\
\text { estate }\end{array}$ & $\begin{array}{l}\text { Marico Kaya } \\
\text { Enterprises } \\
\text { Ltd. }\end{array}$ & 15-Jan-14 & 21.81 & 11.86 & -45.62 & Decline \\
\hline
\end{tabular}




\begin{tabular}{|c|c|c|c|c|c|c|c|c|c|}
\hline 2 & $\begin{array}{l}\text { Avonmore } \\
\text { Capital \& } \\
\text { Mgmt. } \\
\text { Services } \\
\text { Ltd. }\end{array}$ & \multirow{4}{*}{ 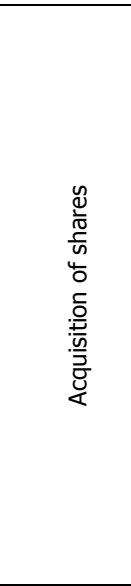 } & $\begin{array}{l}\text { Financial } \\
\text { Services } \\
\text { providers }\end{array}$ & $\begin{array}{l}\text { Almondz } \\
\text { Insurance } \\
\text { Brokers Pvt. } \\
\text { Ltd. }\end{array}$ & 06-Jan-14 & 1.46 & 0.12 & -0.92 & Decline \\
\hline 3 & $\begin{array}{l}\text { Future } \\
\text { Lifestyle } \\
\text { Fashions } \\
\text { Ltd. }\end{array}$ & & $\begin{array}{l}\text { Lifestyle } \\
\text { Fashions }\end{array}$ & $\begin{array}{l}\text { Resource } \\
\text { World Exim } \\
\text { Pvt. Ltd. }\end{array}$ & 03-Jan-14 & 1.07 & 1.51 & 0.41 & Growth \\
\hline 4 & $\begin{array}{l}\text { L \& T } \\
\text { Technology } \\
\text { Services } \\
\text { Ltd. }\end{array}$ & & IT & $\begin{array}{l}\text { Newtime } \\
\text { Infrastructure } \\
\text { Ltd. }\end{array}$ & 14-Jan-14 & 54.46 & 59.36 & 8.99 & Growth \\
\hline 5 & $\begin{array}{l}\text { Oil India } \\
\text { Ltd. }\end{array}$ & & $\begin{array}{l}\text { Petroleum } \\
\text { and } \\
\text { Natural } \\
\text { Gas. }\end{array}$ & $\begin{array}{l}\text { Videocon } \\
\text { Mozambique } \\
\text { Rovuma } 1 \text { Ltd. }\end{array}$ & 07-Jan-14 & 41.76 & 49.59 & 18.75 & Growth \\
\hline \multicolumn{10}{|c|}{ February } \\
\hline 1 & $\begin{array}{l}\text { Relish } \\
\text { Pharmaceuti } \\
\text { cals Ltd. }\end{array}$ & \multirow{3}{*}{ Merger } & $\begin{array}{l}\text { Pharmace } \\
\text { uticals }\end{array}$ & $\begin{array}{l}\text { Divine Heritage } \\
\text { Hotels Pvt. Ltd. }\end{array}$ & 11-Feb-14 & 0.84 & -4.91 & -684.52 & Decline \\
\hline 2 & P V R Ltd. & & Cinemas & $\begin{array}{l}\text { Divine Heritage } \\
\text { Hotels Pvt. Ltd. }\end{array}$ & 14-Feb-14 & 3.3 & 14.16 & 329.09 & Growth \\
\hline 3 & $\begin{array}{l}\text { Lumax D K } \\
\text { Auto } \\
\text { Inds.Ltd. }\end{array}$ & & $\begin{array}{l}\text { Automobil } \\
\text { e }\end{array}$ & $\begin{array}{l}\text { Marico Kaya } \\
\text { Enterprises } \\
\text { Ltd. }\end{array}$ & 27-Feb-14 & 11.02 & 23.51 & 113.34 & Growth \\
\hline 4 & Lupin Ltd. & \multirow{5}{*}{ 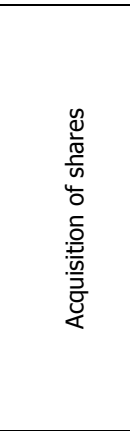 } & $\begin{array}{l}\text { Pharmace } \\
\text { utical }\end{array}$ & $\begin{array}{l}\text { Adani } \\
\text { Transmission } \\
\text { (India) Ltd. }\end{array}$ & 03-Feb-14 & 53.54 & 40.99 & -23.44 & Decline \\
\hline 5 & Lupin Ltd. & & $\begin{array}{l}\text { Pharmace } \\
\text { utical }\end{array}$ & Nanomi B V & 03-Feb-14 & 53.54 & 40.99 & -23.44 & Decline \\
\hline 6 & $\begin{array}{l}\text { Tata Power } \\
\text { Renewable } \\
\text { Energy Ltd. }\end{array}$ & & Power & $\begin{array}{l}\text { M M S } \\
\text { Infrastructure } \\
\text { Ltd. }\end{array}$ & $25-F e b-14$ & 0.19 & 0.46 & 142.11 & Growth \\
\hline 7 & I T C Ltd. & & $\begin{array}{l}\text { Edible } \\
\text { Oils }\end{array}$ & $\begin{array}{l}\text { Oasis Tradelink } \\
\text { Ltd. }\end{array}$ & 06-Feb-14 & 12.05 & 11.09 & -7.97 & Decline \\
\hline 8 & Omaxe Ltd. & & Builders & $\begin{array}{l}\text { P P Devcon } \\
\text { Pvt. Ltd. }\end{array}$ & 07-Feb-14 & 2.18 & 3.3 & 51.38 & Growth \\
\hline \multicolumn{10}{|c|}{ March } \\
\hline 1 & $\begin{array}{l}\text { Ravindra } \\
\text { Energy } \\
\text { Ltd. }\end{array}$ & \multirow[t]{2}{*}{ Merger } & Power & $\begin{array}{l}\text { Shree Renuka } \\
\text { Energy Ltd. } \\
\text { [Merged] }\end{array}$ & 18-Mar-14 & 0.03 & 0.02 & -33.33 & Decline \\
\hline 2 & $\begin{array}{l}\text { Ashok } \\
\text { Leyland } \\
\text { Ltd. }\end{array}$ & & $\begin{array}{l}\text { Automobil } \\
\text { e }\end{array}$ & $\begin{array}{l}\text { Divine Heritage } \\
\text { Hotels Pvt. Ltd. }\end{array}$ & 27-Mar-14 & 1.2 & 0.11 & -90.83 & Decline \\
\hline 3 & $\begin{array}{l}\text { Power } \\
\text { Grid } \\
\text { Corpn. Of } \\
\text { India Ltd. }\end{array}$ & 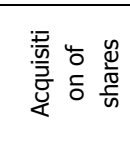 & Power & $\begin{array}{l}\text { Unchahar } \\
\text { Transmission } \\
\text { Ltd. }\end{array}$ & 24-Mar-14 & 9.52 & 9.36 & -1.68 & Decline \\
\hline
\end{tabular}




\begin{tabular}{|c|c|c|c|c|c|c|c|c|}
\hline 4 & $\begin{array}{l}\text { Oil India } \\
\text { Ltd. }\end{array}$ & $\begin{array}{l}\text { Petroleum } \\
\text { and } \\
\text { Natural } \\
\text { Gas. }\end{array}$ & $\begin{array}{l}\text { Indian Oil } \\
\text { Corpn. Ltd. }\end{array}$ & 14-Mar-14 & 41.76 & 49.59 & 18.75 & Growth \\
\hline 5 & $\begin{array}{l}\text { R P P } \\
\text { Infra } \\
\text { Projects } \\
\text { Ltd. }\end{array}$ & $\begin{array}{l}\text { Infrastruct } \\
\text { ure }\end{array}$ & $\begin{array}{l}\text { Greatful } \\
\text { Mercantile Pvt. } \\
\text { Ltd. }\end{array}$ & 28-Mar-14 & 7.64 & 5.17 & -32.33 & Decline \\
\hline 6 & $\begin{array}{l}\text { R P P } \\
\text { Infra } \\
\text { Projects } \\
\text { Ltd. }\end{array}$ & $\begin{array}{l}\text { Infrastruct } \\
\text { ure }\end{array}$ & $\begin{array}{l}\text { Birdhi Chand } \\
\text { Pannalal } \\
\text { Agencies Ltd. }\end{array}$ & 28-Mar-14 & 7.64 & 5.17 & -32.33 & Decline \\
\hline 7 & $\begin{array}{l}\text { Sterlite } \\
\text { Grid Ltd. }\end{array}$ & $\begin{array}{l}\text { Telecom \& } \\
\text { Power Ltd. }\end{array}$ & $\begin{array}{l}\text { Rapp } \\
\text { Transmission } \\
\text { Co. Ltd. }\end{array}$ & 12-Mar-14 & 2.14 & 1.28 & -40.19 & Decline \\
\hline 8 & $\begin{array}{l}\text { Surana } \\
\text { Telecom } \\
\text { \& Power } \\
\text { Ltd. }\end{array}$ & $\begin{array}{l}\text { Telecom \& } \\
\text { Power Ltd. }\end{array}$ & $\begin{array}{l}\text { Surana Solar } \\
\text { Systems Pvt. } \\
\text { Ltd. }\end{array}$ & 21-Mar-14 & 0.19 & 0.07 & -63.16 & Decline \\
\hline 9 & U P L Ltd. & Chemical & $\begin{array}{l}\text { U P L Do Brasil } \\
\text { Industria E } \\
\text { Comercio De } \\
\text { Insumos } \\
\text { Agropecuarios } \\
\text { S A }\end{array}$ & 04-Mar-14 & 10.81 & 9.45 & -12.58 & Decline \\
\hline
\end{tabular}

Source: From Annual Report with Authors' calculation 


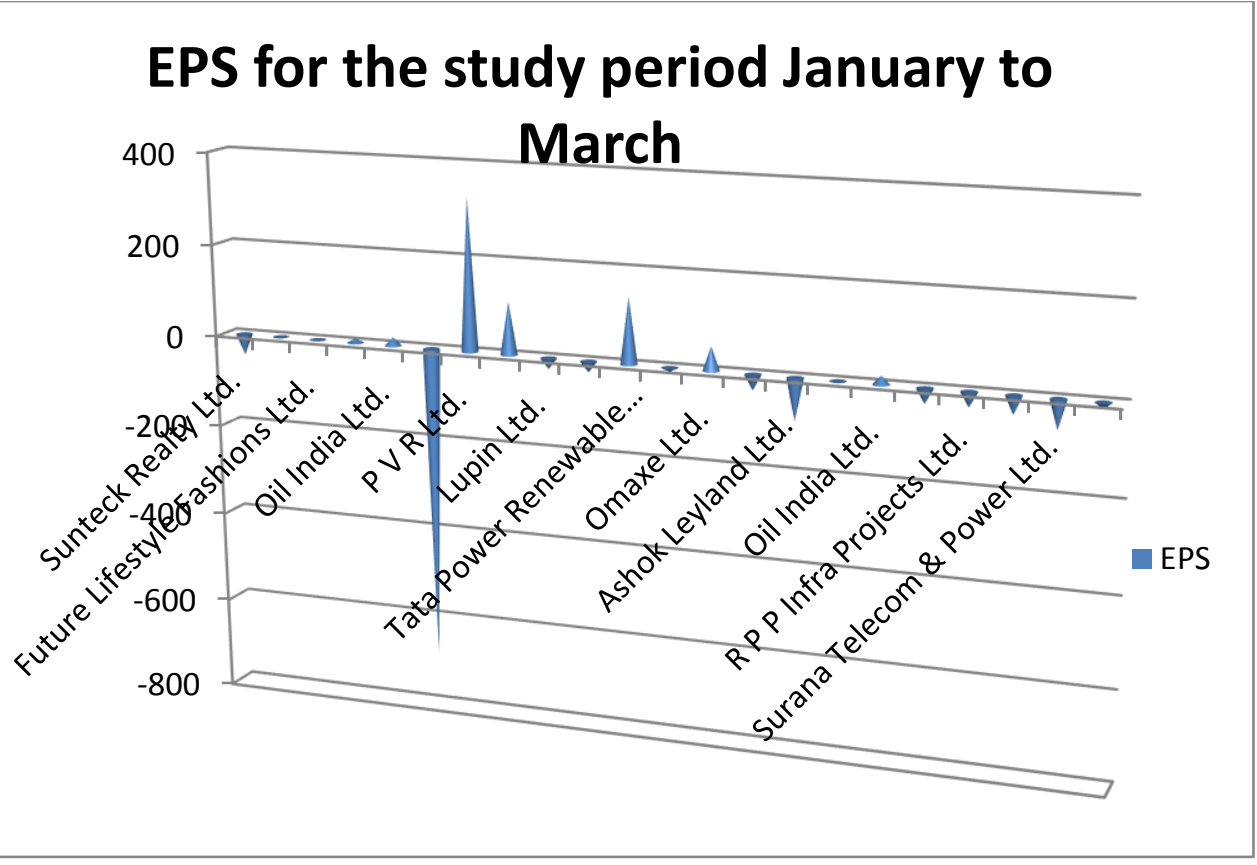

\section{Interpretation:}

The above table shows that the EPS calculations of 5 M\&A firms for the month of January 2014 show a major growth perspective transaction over Merging and Acquiring firms. In the month of February 2014, the 8 acquiring firms show a growth as well as decline perspective value. For the month of March 2014, the 9 acquiring firms shows a major decline over the transaction made on Merging and Acquiring. It reveals that the EPS growth percentage calculation shows a common decline trend in the Q1 of 2014. 
6. Table showing NETWORTH calculation:

\begin{tabular}{|c|c|c|c|c|c|c|c|c|c|}
\hline \multirow[b]{2}{*}{$\frac{o}{n}$} & \multirow{2}{*}{ 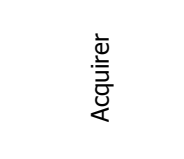 } & \multirow{2}{*}{ 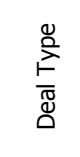 } & \multirow[b]{2}{*}{ 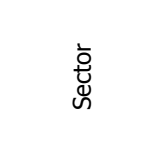 } & \multirow{2}{*}{ 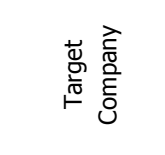 } & \multirow{2}{*}{ 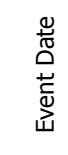 } & \multicolumn{4}{|c|}{ NETWORTH } \\
\hline & & & & & & $\begin{array}{l}\text { BM } \\
\text { (Rs.in } \\
\text { Crore) }\end{array}$ & $\begin{array}{l}\text { AM(Rs. } \\
\text { In Crore) }\end{array}$ & $\begin{array}{l}\text { Networth } \\
\text { Growth } \\
\text { in } \%\end{array}$ & $\begin{array}{l}\text { Growth } \\
\text { /Decline }\end{array}$ \\
\hline \multicolumn{10}{|c|}{ January } \\
\hline 1 & $\begin{array}{l}\text { Sunteck Realty } \\
\text { Ltd. }\end{array}$ & Merger & Real estate & $\begin{array}{l}\text { Marico Kaya } \\
\text { Enterprises } \\
\text { Ltd. }\end{array}$ & $\begin{array}{l}15- \\
\text { Jan- } \\
14\end{array}$ & $\begin{array}{l}1470.28 \\
2\end{array}$ & 547.747 & 168.42 & Growth \\
\hline 2 & $\begin{array}{l}\text { Avonmore } \\
\text { Capital \& } \\
\text { Mgmt. Services } \\
\text { Ltd. }\end{array}$ & \multirow{4}{*}{$\begin{array}{l}\text { U } \\
\frac{1}{0} \\
\frac{1}{5} \\
\frac{4}{0} \\
\overline{0} \\
\frac{0}{+0} \\
\frac{5}{3} \\
\frac{0}{4}\end{array}$} & $\begin{array}{l}\text { Financial } \\
\text { Services } \\
\text { providers }\end{array}$ & $\begin{array}{l}\text { Almondz } \\
\text { Insurance } \\
\text { Brokers Pvt. } \\
\text { Ltd. }\end{array}$ & $\begin{array}{l}06- \\
\text { Jan- } \\
14\end{array}$ & 69.46 & 58.54 & 18.66 & Growth \\
\hline 3 & $\begin{array}{l}\text { Future Lifestyle } \\
\text { Fashions Ltd. }\end{array}$ & & $\begin{array}{l}\text { Lifestyle } \\
\text { Fashions }\end{array}$ & $\begin{array}{l}\text { Resource } \\
\text { World Exim } \\
\text { Pvt. Ltd. }\end{array}$ & $\begin{array}{l}03- \\
\text { Jan- } \\
14\end{array}$ & $\begin{array}{l}1,569.7 \\
2\end{array}$ & $1,291.32$ & 21.56 & Growth \\
\hline 4 & $\begin{array}{l}\text { L \& T } \\
\text { Technology } \\
\text { Services Ltd. }\end{array}$ & & IT & $\begin{array}{l}\text { Newtime } \\
\text { Infrastructure } \\
\text { Ltd. }\end{array}$ & $\begin{array}{l}14- \\
\text { Jan- } \\
14\end{array}$ & $\begin{array}{l}37,084 . \\
6\end{array}$ & $33,661.8$ & 10.17 & Growth \\
\hline 5 & Oil India Ltd. & & $\begin{array}{l}\text { Petroleum } \\
\text { and Natural } \\
\text { Gas. }\end{array}$ & $\begin{array}{l}\text { Videocon } \\
\text { Mozambique } \\
\text { Rovuma } 1 \\
\text { Ltd. }\end{array}$ & $\begin{array}{l}07- \\
\text { Jan- } \\
14\end{array}$ & $\begin{array}{l}21,514 . \\
31\end{array}$ & $\begin{array}{l}20,708.1 \\
8\end{array}$ & 3.89 & Growth \\
\hline \multicolumn{10}{|c|}{ February } \\
\hline 1 & $\begin{array}{l}\text { Relish } \\
\text { Pharmaceutical } \\
\text { s Ltd. }\end{array}$ & \multirow{3}{*}{ Merger } & $\begin{array}{l}\text { Pharmaceuti } \\
\text { cals }\end{array}$ & $\begin{array}{l}\text { Divine } \\
\text { Heritage } \\
\text { Hotels Pvt. } \\
\text { Ltd. }\end{array}$ & $\begin{array}{l}11- \\
\text { Feb- } \\
14\end{array}$ & 13.954 & 13.823 & 0.95 & Growth \\
\hline 2 & P V R Ltd. & & Cinemas & $\begin{array}{l}\text { Divine } \\
\text { Heritage } \\
\text { Hotels Pvt. } \\
\text { Ltd. }\end{array}$ & $\begin{array}{l}14- \\
\text { Feb- } \\
14\end{array}$ & $3,983.7$ & $3,912.7$ & 1.81 & Growth \\
\hline 3 & $\begin{array}{l}\text { Lumax D K } \\
\text { Auto Inds. Ltd. }\end{array}$ & & Automobile & $\begin{array}{l}\text { Marico Kaya } \\
\text { Enterprises } \\
\text { Ltd. }\end{array}$ & $\begin{array}{l}27- \\
\text { Feb- } \\
14\end{array}$ & 123.792 & 103.366 & 19.76 & Growth \\
\hline 4 & Lupin Ltd. & \multirow{4}{*}{ 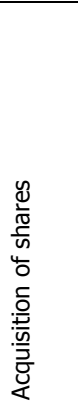 } & $\begin{array}{l}\text { Pharmaceuti } \\
\text { cal }\end{array}$ & $\begin{array}{l}\text { Adani } \\
\text { Transmission } \\
\text { (India) Ltd. }\end{array}$ & $\begin{array}{l}03- \\
\text { Feb- } \\
14\end{array}$ & $\begin{array}{l}8,874.0 \\
6\end{array}$ & $6,931.57$ & 28.02 & Growth \\
\hline 5 & Lupin Ltd. & & $\begin{array}{l}\text { Pharmaceuti } \\
\text { cal }\end{array}$ & Nanomi B V & $\begin{array}{l}03- \\
\text { Feb- } \\
14\end{array}$ & $\begin{array}{l}8,874.0 \\
6\end{array}$ & $6,931.57$ & 28.02 & Growth \\
\hline 6 & $\begin{array}{l}\text { Tata Power } \\
\text { Renewable } \\
\text { Energy Ltd. }\end{array}$ & & Power & $\begin{array}{l}\text { M M S } \\
\text { Infrastructure } \\
\text { Ltd. }\end{array}$ & $\begin{array}{l}25- \\
\text { Feb- } \\
14\end{array}$ & 500.307 & 201.416 & 148.39 & Growth \\
\hline 7 & I T C Ltd. & & Edible Oils & $\begin{array}{l}\text { Oasis } \\
\text { Tradelink Ltd. }\end{array}$ & $\begin{array}{l}06- \\
\text { Feb- } \\
14\end{array}$ & $\begin{array}{l}30735.6 \\
9\end{array}$ & 26262.02 & 17.03 & Growth \\
\hline
\end{tabular}




\begin{tabular}{|c|c|c|c|c|c|c|c|c|c|}
\hline 8 & Omaxe Ltd. & & Builders & $\begin{array}{l}\text { P P Devcon } \\
\text { Pvt. Ltd. }\end{array}$ & $\begin{array}{l}07- \\
\text { Feb- } \\
14 \\
\end{array}$ & $\begin{array}{l}1,911.6 \\
1\end{array}$ & $1,882.95$ & 1.52 & Growth \\
\hline \multicolumn{10}{|c|}{ March } \\
\hline 1 & $\begin{array}{l}\text { Ravindra } \\
\text { Energy Ltd. }\end{array}$ & & Power & $\begin{array}{l}\text { Shree Renuka } \\
\text { Energy Ltd. } \\
\text { [Merged] }\end{array}$ & $\begin{array}{l}18- \\
\text { Mar- } \\
14\end{array}$ & 204.121 & 203.052 & 0.53 & Growth \\
\hline 2 & $\begin{array}{l}\text { Ashok Leyland } \\
\text { Ltd. }\end{array}$ & Merger & Automobile & $\begin{array}{l}\text { Divine } \\
\text { Heritage } \\
\text { Hotels Pvt. } \\
\text { Ltd. }\end{array}$ & $\begin{array}{l}27- \\
\text { Mar- } \\
14\end{array}$ & $\begin{array}{l}5,118.7 \\
0\end{array}$ & $4,447.89$ & 15.08 & Growth \\
\hline 3 & $\begin{array}{l}\text { Power Grid } \\
\text { Corpn. Of India } \\
\text { Ltd. }\end{array}$ & \multirow{7}{*}{ 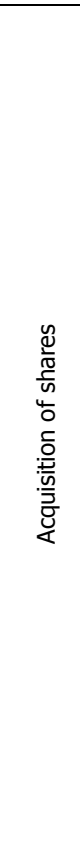 } & Power & $\begin{array}{l}\text { Unchahar } \\
\text { Transmission } \\
\text { Ltd. }\end{array}$ & $\begin{array}{l}24- \\
\text { Mar- } \\
14\end{array}$ & $\begin{array}{l}38166.5 \\
9\end{array}$ & 34459.63 & 10.76 & Growth \\
\hline 4 & Oil India Ltd. & & $\begin{array}{l}\text { Petroleum } \\
\text { and Natural } \\
\text { Gas. }\end{array}$ & $\begin{array}{l}\text { Indian Oil } \\
\text { Corpn. Ltd. }\end{array}$ & $\begin{array}{l}14- \\
\text { Mar- } \\
14\end{array}$ & $\begin{array}{l}21514.3 \\
1\end{array}$ & 20708.18 & 3.89 & Growth \\
\hline 5 & $\begin{array}{l}\text { R P P Infra } \\
\text { Projects Ltd. }\end{array}$ & & $\begin{array}{l}\text { Infrastructu } \\
\text { re }\end{array}$ & $\begin{array}{l}\text { Greatful } \\
\text { Mercantile } \\
\text { Pvt. Ltd. }\end{array}$ & $\begin{array}{l}28- \\
\text { Mar- } \\
14\end{array}$ & 135.85 & 119.97 & 13.24 & Growth \\
\hline 6 & $\begin{array}{l}\text { R P P Infra } \\
\text { Projects Ltd. }\end{array}$ & & $\begin{array}{l}\text { Infrastructu } \\
\text { re }\end{array}$ & $\begin{array}{l}\text { Birdhi Chand } \\
\text { Pannalal } \\
\text { Agencies Ltd. }\end{array}$ & $\begin{array}{l}28- \\
\text { Mar- } \\
14\end{array}$ & 135.85 & 119.97 & 13.24 & Growth \\
\hline 7 & $\begin{array}{l}\text { Sterlite Grid } \\
\text { Ltd. }\end{array}$ & & $\begin{array}{l}\text { Telecom \& } \\
\text { Power Ltd. }\end{array}$ & $\begin{array}{l}\text { Rapp } \\
\text { Transmission } \\
\text { Co. Ltd. }\end{array}$ & $\begin{array}{l}12- \\
\text { Mar- } \\
14\end{array}$ & $\begin{array}{l}1,242.6 \\
5\end{array}$ & $1,218.29$ & 1.99 & Growth \\
\hline 8 & $\begin{array}{l}\text { Surana } \\
\text { Telecom \& } \\
\text { Power Ltd. }\end{array}$ & & $\begin{array}{l}\text { Telecom \& } \\
\text { Power Ltd. }\end{array}$ & $\begin{array}{l}\text { Surana Solar } \\
\text { Systems Pvt. } \\
\text { Ltd. }\end{array}$ & $\begin{array}{l}21- \\
\text { Mar- } \\
14 \\
\end{array}$ & 59.814 & 60.136 & -0.53 & Decline \\
\hline 9 & U P L Ltd. & & Chemical & $\begin{array}{l}\text { U P L Do } \\
\text { Brasil } \\
\text { Industria E } \\
\text { Comercio De } \\
\text { Insumos } \\
\text { Agropecuario } \\
\text { S S A }\end{array}$ & $\begin{array}{l}04- \\
\text { Mar- } \\
14\end{array}$ & $\begin{array}{l}3,524.0 \\
8\end{array}$ & 3,308 & 6.54 & Growth \\
\hline
\end{tabular}

\section{Source: From Annual Report with Authors' calculation}




\section{Networth for the study period January to March}

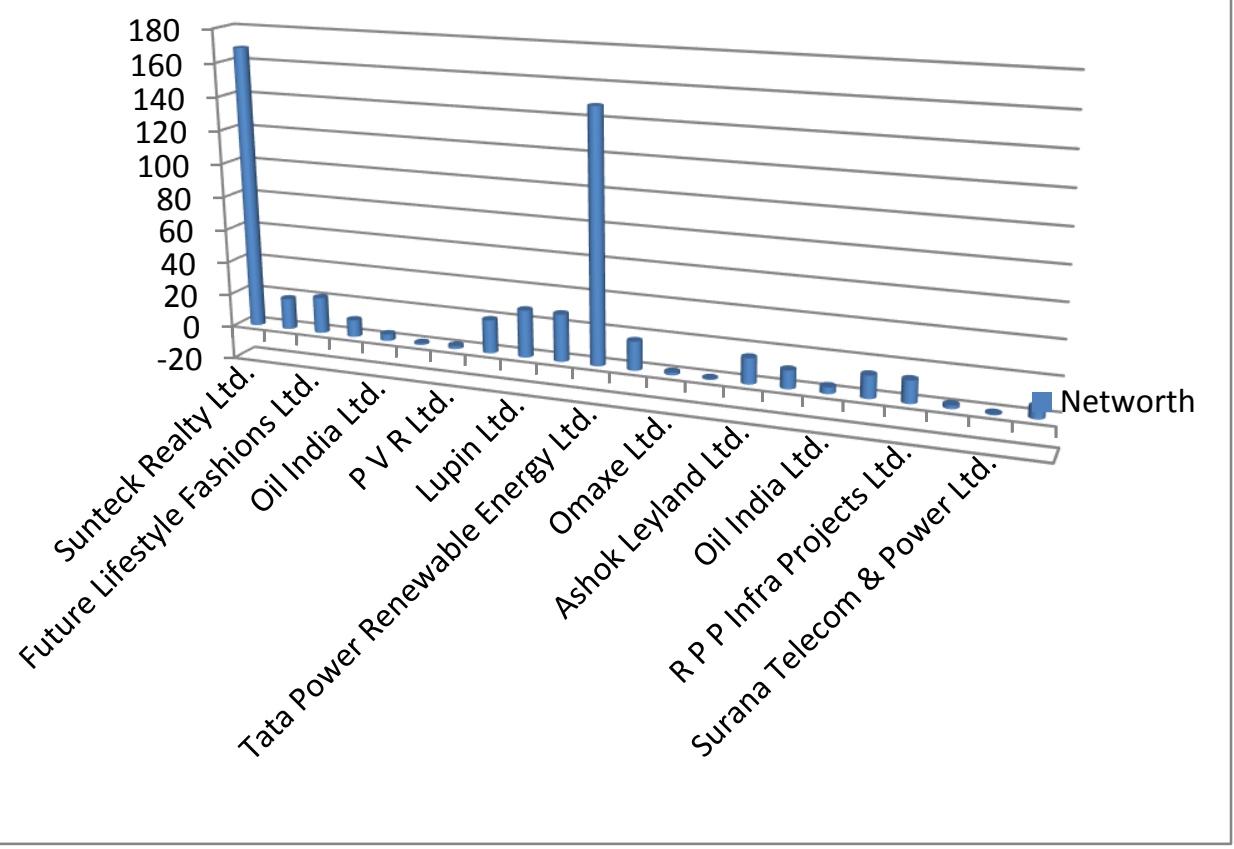

\section{Interpretation:}

The above table reveals that for 3months NETWORTH calculations, which have a major growth perspective in common over Merging and Acquiring firms. In the month of March 2014, only one transaction has hit decline. It reveals that the NETWORTH shows a growth stage over the transaction made in the 3 consecutive months of 2014. 
7. Table showing DEBT EQUITY RATIO calculation:

\begin{tabular}{|c|c|c|c|c|c|c|c|c|c|}
\hline \multirow[b]{2}{*}{$\frac{\circ}{n}$} & \multirow[b]{2}{*}{ 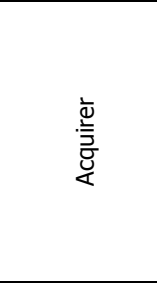 } & \multirow{2}{*}{ 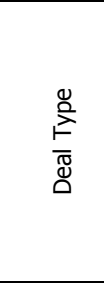 } & \multirow[b]{2}{*}{ 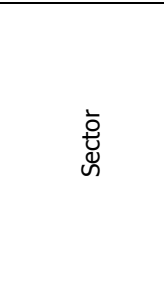 } & \multirow{2}{*}{ 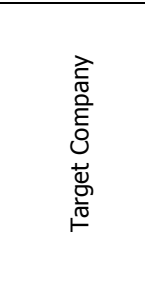 } & \multirow{2}{*}{ 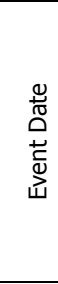 } & \multicolumn{4}{|c|}{ DEBT EQUITY RATIO } \\
\hline & & & & & & $\begin{array}{l}\text { BM } \\
\text { (Rs.in } \\
\text { Crore) }\end{array}$ & $\begin{array}{l}\text { AM(Rs. } \\
\text { In } \\
\text { Crore) }\end{array}$ & $\begin{array}{l}\text { Debt } \\
\text { Equity } \\
\text { Growt } \\
\mathrm{h} \text { in } \\
\%\end{array}$ & $\begin{array}{l}\text { Growt } \\
\mathrm{h} \\
\text { /Declin } \\
\mathrm{e}\end{array}$ \\
\hline \multicolumn{10}{|c|}{ January } \\
\hline 1 & $\begin{array}{l}\text { Sunteck } \\
\text { Realty Ltd. }\end{array}$ & Merger & Real estate & $\begin{array}{l}\text { Marico Kaya } \\
\text { Enterprises } \\
\text { Ltd. }\end{array}$ & $\begin{array}{l}15- \\
\text { Jan } \\
-14\end{array}$ & $\begin{array}{l}2257.38 \\
3\end{array}$ & 1155.55 & 95.35 & $\begin{array}{l}\text { Growt } \\
h\end{array}$ \\
\hline 2 & $\begin{array}{l}\text { Avonmore } \\
\text { Capital \& } \\
\text { Mgmt. } \\
\text { Services Ltd. }\end{array}$ & \multirow{4}{*}{$\begin{array}{l}\text { Acquisiti } \\
\text { on of } \\
\text { shares }\end{array}$} & $\begin{array}{l}\text { Financial } \\
\text { Services } \\
\text { providers }\end{array}$ & $\begin{array}{l}\text { Almondz } \\
\text { Insurance } \\
\text { Brokers Pvt. } \\
\text { Ltd. }\end{array}$ & $\begin{array}{l}06- \\
\text { Jan } \\
-14\end{array}$ & 139.263 & 118.832 & 17.19 & $\begin{array}{l}\text { Growt } \\
\text { h }\end{array}$ \\
\hline 3 & $\begin{array}{l}\text { Future } \\
\text { Lifestyle } \\
\text { Fashions Ltd. }\end{array}$ & & $\begin{array}{l}\text { Lifestyle } \\
\text { Fashions }\end{array}$ & $\begin{array}{l}\text { Resource } \\
\text { World Exim } \\
\text { Pvt. Ltd. }\end{array}$ & $\begin{array}{l}03- \\
\text { Jan } \\
-14\end{array}$ & 5518.59 & 5231.65 & 5.48 & $\begin{array}{l}\text { Growt } \\
\text { h }\end{array}$ \\
\hline 4 & $\begin{array}{l}\text { L \& T } \\
\text { Technology } \\
\text { Services Ltd. }\end{array}$ & & IT & $\begin{array}{l}\text { Newtime } \\
\text { Infrastructu } \\
\text { re Ltd. }\end{array}$ & $\begin{array}{l}14- \\
\text { Jan } \\
-14 \\
\end{array}$ & $\begin{array}{l}123988 . \\
4\end{array}$ & $\begin{array}{l}111966 . \\
4\end{array}$ & 10.74 & $\begin{array}{l}\text { Growt } \\
h\end{array}$ \\
\hline 5 & Oil India Ltd. & & $\begin{array}{l}\text { Petroleum } \\
\text { and Natural } \\
\text { Gas. }\end{array}$ & $\begin{array}{l}\text { Videocon } \\
\text { Mozambiqu } \\
\text { e Rovuma } 1 \\
\text { Ltd. }\end{array}$ & $\begin{array}{l}07- \\
\text { Jan } \\
-14\end{array}$ & $\begin{array}{l}57741.4 \\
3\end{array}$ & $\begin{array}{l}55582.6 \\
3\end{array}$ & 3.88 & $\begin{array}{l}\text { Growt } \\
h\end{array}$ \\
\hline \multicolumn{10}{|c|}{ February } \\
\hline 1 & $\begin{array}{l}\text { Relish } \\
\text { Pharmaceutic } \\
\text { als Ltd. }\end{array}$ & \multirow{3}{*}{ Merger } & $\begin{array}{l}\text { Pharmaceutic } \\
\text { als }\end{array}$ & $\begin{array}{l}\text { Divine } \\
\text { Heritage } \\
\text { Hotels Pvt. } \\
\text { Ltd. }\end{array}$ & $\begin{array}{l}11- \\
\text { Feb } \\
-14\end{array}$ & 32.265 & 32.065 & 0.62 & $\begin{array}{l}\text { Growt } \\
h\end{array}$ \\
\hline 2 & P V R Ltd. & & Cinemas & $\begin{array}{l}\text { Divine } \\
\text { Heritage } \\
\text { Hotels Pvt. } \\
\text { Ltd. }\end{array}$ & $\begin{array}{l}14- \\
\text { Feb } \\
-14\end{array}$ & 1763.04 & 1621.58 & 8.72 & $\begin{array}{l}\text { Growt } \\
h\end{array}$ \\
\hline 3 & $\begin{array}{l}\text { Lumax D K } \\
\text { Auto Inds. } \\
\text { Ltd. }\end{array}$ & & Automobile & $\begin{array}{l}\text { Marico Kaya } \\
\text { Enterprises } \\
\text { Ltd. }\end{array}$ & $\begin{array}{l}27- \\
\text { Feb } \\
-14\end{array}$ & 605.048 & 576.792 & 4.89 & $\begin{array}{l}\text { Growt } \\
h\end{array}$ \\
\hline 4 & Lupin Ltd. & & $\begin{array}{l}\text { Pharmaceutic } \\
\text { al }\end{array}$ & $\begin{array}{l}\text { Adani } \\
\text { Transmissio } \\
\text { n (India) } \\
\text { Ltd. }\end{array}$ & $\begin{array}{l}03- \\
\text { Feb } \\
-14\end{array}$ & 22011.8 & 17137.6 & 28.44 & $\begin{array}{l}\text { Growt } \\
h\end{array}$ \\
\hline
\end{tabular}




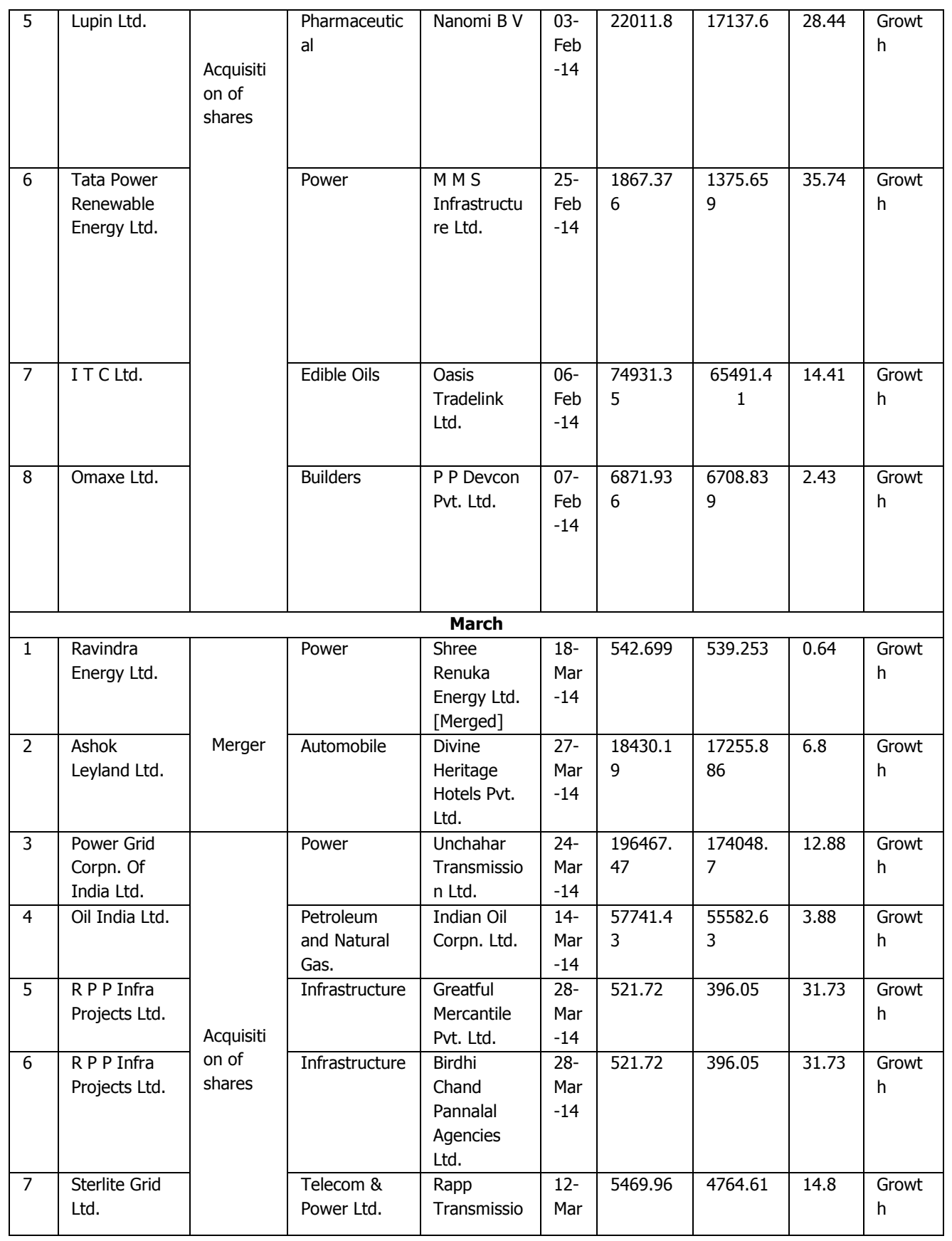




\begin{tabular}{|c|c|c|c|c|c|c|c|c|}
\hline & & & n Co. Ltd. & -14 & & & & \\
\hline 8 & $\begin{array}{l}\text { Surana } \\
\text { Telecom \& } \\
\text { Power Ltd. }\end{array}$ & $\begin{array}{l}\text { Telecom \& } \\
\text { Power Ltd. }\end{array}$ & $\begin{array}{l}\text { Surana } \\
\text { Solar } \\
\text { Systems } \\
\text { Pvt. Ltd. }\end{array}$ & $\begin{array}{l}21- \\
\text { Mar } \\
-14\end{array}$ & 154.842 & 156.444 & -1.02 & $\begin{array}{l}\text { Declin } \\
\text { e }\end{array}$ \\
\hline 9 & U P L Ltd. & Chemical & $\begin{array}{l}\text { U P L Do } \\
\text { Brasil } \\
\text { Industria E } \\
\text { Comercio } \\
\text { De Insumos } \\
\text { Agropecuari } \\
\text { os S A }\end{array}$ & $\begin{array}{l}04- \\
\text { Mar } \\
-14\end{array}$ & $\begin{array}{l}11163.3 \\
9\end{array}$ & $\begin{array}{l}10390.1 \\
6\end{array}$ & 7.44 & $\begin{array}{l}\text { Growt } \\
h\end{array}$ \\
\hline
\end{tabular}

Source: From Annual Report with Authors' calculation

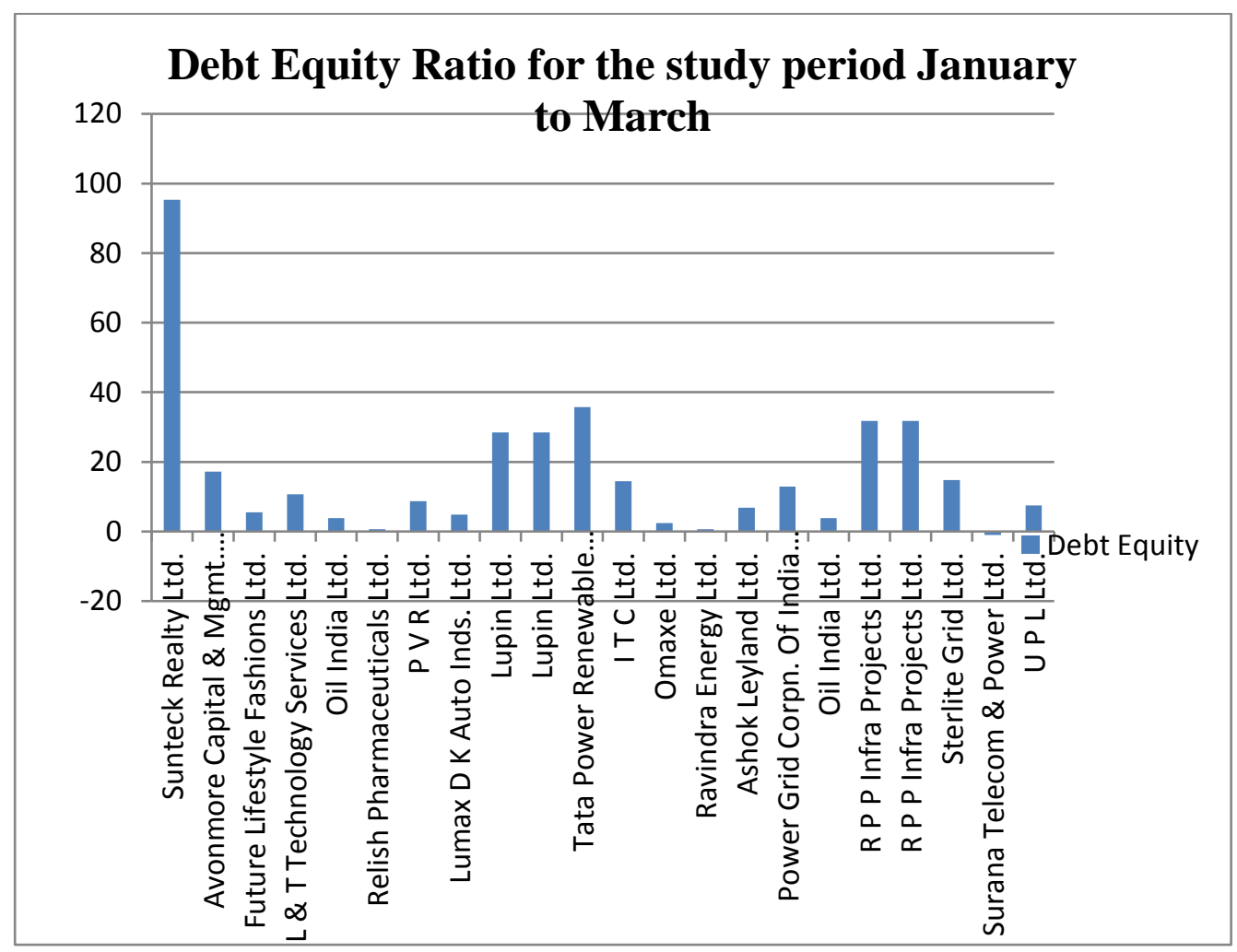




\section{Interpretation:}

From the above table, we can infer that except for one transaction in the entire study period, all other company's Debt Equity Ratios have shown a growing/increasing trend.

\section{Table showing MARKET VALUE OF COMPANY calculation:}

\begin{tabular}{|c|c|c|c|c|c|c|c|c|c|}
\hline \multirow[b]{2}{*}{$\frac{\circ}{n}$} & \multirow[b]{2}{*}{ 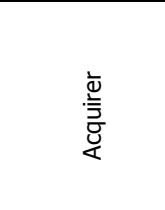 } & \multirow[b]{2}{*}{$\frac{\stackrel{\Xi}{\beth}}{\frac{\mathscr{Z}}{\mathscr{D}}}$} & \multirow[b]{2}{*}{ 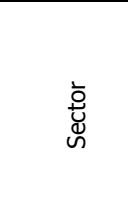 } & \multirow[b]{2}{*}{ 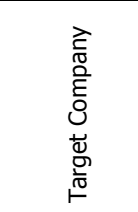 } & \multirow[b]{2}{*}{ 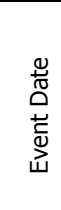 } & \multicolumn{4}{|c|}{ MARKET VALUE OF COMPANY } \\
\hline & & & & & & $\begin{array}{l}\text { BM } \\
\text { (Rs.in } \\
\text { Crore) }\end{array}$ & $\begin{array}{l}\text { AM(Rs. } \\
\text { In Crore) }\end{array}$ & $\begin{array}{l}\text { Growth } \\
\text { in } \%\end{array}$ & $\begin{array}{l}\text { Growth } \\
\text { /Decline }\end{array}$ \\
\hline \multicolumn{10}{|c|}{ January } \\
\hline 1 & $\begin{array}{l}\text { Sunteck } \\
\text { Realty Ltd. }\end{array}$ & Merger & $\begin{array}{l}\text { Real } \\
\text { estate }\end{array}$ & $\begin{array}{l}\text { Marico Kaya } \\
\text { Enterprises } \\
\text { Ltd. }\end{array}$ & $\begin{array}{l}15- \\
\text { Jan- } \\
14\end{array}$ & 1960.70 & 1696.55 & -13.47 & Decline \\
\hline 2 & $\begin{array}{l}\text { Avonmore } \\
\text { Capital \& } \\
\text { Mgmt. } \\
\text { Services Ltd. }\end{array}$ & $\begin{array}{l}\text { Acquisition } \\
\text { of shares }\end{array}$ & $\begin{array}{l}\text { Financial } \\
\text { Services } \\
\text { providers }\end{array}$ & $\begin{array}{l}\text { Almondz } \\
\text { Insurance } \\
\text { Brokers Pvt. } \\
\text { Ltd. }\end{array}$ & $\begin{array}{l}06- \\
\text { Jan- } \\
14\end{array}$ & 39.13 & 48.42 & 23.75 & Growth \\
\hline 3 & $\begin{array}{l}\text { Future } \\
\text { Lifestyle } \\
\text { Fashions Ltd. }\end{array}$ & \multirow[b]{3}{*}{$\begin{array}{l}\text { Acquisition } \\
\text { of shares }\end{array}$} & $\begin{array}{l}\text { Lifestyle } \\
\text { Fashions }\end{array}$ & $\begin{array}{l}\text { Resource } \\
\text { World Exim } \\
\text { Pvt. Ltd. }\end{array}$ & $\begin{array}{l}03- \\
\text { Jan- } \\
14 \\
\end{array}$ & 2.02 & 2.09 & 3.62 & Growth \\
\hline 4 & $\begin{array}{l}\mathrm{L} \& \mathrm{~T} \\
\text { Technology } \\
\text { Services Ltd. }\end{array}$ & & IT & $\begin{array}{l}\text { Newtime } \\
\text { Infrastructu } \\
\text { re Ltd. }\end{array}$ & $\begin{array}{l}14- \\
\text { Jan- } \\
14\end{array}$ & $\begin{array}{l}99547.9 \\
9\end{array}$ & 92719.88 & -6.86 & Decline \\
\hline 5 & Oil India Ltd. & & $\begin{array}{l}\text { Petroleum } \\
\text { and } \\
\text { Natural } \\
\text { Gas. }\end{array}$ & $\begin{array}{l}\text { Videocon } \\
\text { Mozambiqu } \\
\text { e Rovuma } 1 \\
\text { Ltd. }\end{array}$ & $\begin{array}{l}07- \\
\text { Jan- } \\
14\end{array}$ & $\begin{array}{l}28211.8 \\
8\end{array}$ & 28343.43 & 0.47 & Growth \\
\hline \multicolumn{10}{|c|}{ February } \\
\hline 1 & $\begin{array}{l}\text { Relish } \\
\text { Pharmaceutic } \\
\text { als Ltd. }\end{array}$ & \multirow{3}{*}{ Merger } & $\begin{array}{l}\text { Pharmace } \\
\text { uticals }\end{array}$ & $\begin{array}{l}\text { Divine } \\
\text { Heritage } \\
\text { Hotels Pvt. } \\
\text { Ltd. }\end{array}$ & $\begin{array}{l}\text { 11- } \\
\text { Feb- } \\
14\end{array}$ & 1.19 & 1.85 & 55.73 & Growth \\
\hline 2 & P V R Ltd. & & Cinemas & $\begin{array}{l}\text { Divine } \\
\text { Heritage } \\
\text { Hotels Pvt. } \\
\text { Ltd. }\end{array}$ & 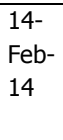 & 2508.37 & 2230.83 & -11.06 & Decline \\
\hline 3 & $\begin{array}{l}\text { Lumax D K } \\
\text { Auto Inds. }\end{array}$ & & $\begin{array}{l}\text { Automobil } \\
\text { e }\end{array}$ & $\begin{array}{l}\text { Marico Kaya } \\
\text { Enterprises }\end{array}$ & $\begin{array}{l}27- \\
\text { Feb- }\end{array}$ & 139.45 & 131.44 & -5.75 & Decline \\
\hline
\end{tabular}




\begin{tabular}{|c|c|c|c|c|c|c|c|c|c|}
\hline & Ltd. & & & Ltd. & 14 & & & & \\
\hline 4 & Lupin Ltd. & \multirow{5}{*}{$\begin{array}{l}\text { Acquisition } \\
\text { of shares }\end{array}$} & $\begin{array}{l}\text { Pharmace } \\
\text { utical }\end{array}$ & $\begin{array}{l}\text { Adani } \\
\text { Transmissio } \\
\text { n (India) } \\
\text { Ltd. }\end{array}$ & $\begin{array}{l}03- \\
\text { Feb- } \\
14\end{array}$ & $\begin{array}{l}40967.8 \\
0\end{array}$ & 41615.88 & 1.58 & Growth \\
\hline 5 & Lupin Ltd. & & $\begin{array}{l}\text { Pharmace } \\
\text { utical }\end{array}$ & Nanomi B V & $\begin{array}{l}03- \\
\text { Feb- } \\
14\end{array}$ & $\begin{array}{l}40967.8 \\
0\end{array}$ & 41615.88 & 1.58 & Growth \\
\hline 6 & $\begin{array}{l}\text { Tata Power } \\
\text { Renewable } \\
\text { Energy Ltd. }\end{array}$ & & Power & $\begin{array}{l}\text { M M S } \\
\text { Infrastructu } \\
\text { re Ltd. }\end{array}$ & $\begin{array}{l}25- \\
\text { Feb- } \\
14\end{array}$ & 1854.50 & 1493.91 & -19.44 & Decline \\
\hline 7 & I T C Ltd. & & $\begin{array}{l}\text { Edible } \\
\text { Oils }\end{array}$ & $\begin{array}{l}\text { Oasis } \\
\text { Tradelink } \\
\text { Ltd. }\end{array}$ & $\begin{array}{l}06- \\
\text { Feb- } \\
14\end{array}$ & $\begin{array}{l}257072 . \\
81\end{array}$ & $\begin{array}{l}255355.7 \\
8\end{array}$ & -0.67 & Decline \\
\hline 8 & Omaxe Ltd. & & Builders & $\begin{array}{l}\text { P P Devcon } \\
\text { Pvt. Ltd. }\end{array}$ & $\begin{array}{l}07- \\
\text { Feb- } \\
14\end{array}$ & 3119.15 & 3104.67 & -0.46 & Decline \\
\hline \multicolumn{10}{|c|}{ March } \\
\hline 1 & $\begin{array}{l}\text { Ravindra } \\
\text { Energy Ltd. }\end{array}$ & \multirow[b]{2}{*}{ Merger } & Power & $\begin{array}{l}\text { Shree } \\
\text { Renuka } \\
\text { Energy Ltd. } \\
\text { [Merged] }\end{array}$ & $\begin{array}{l}18- \\
\text { Mar- } \\
14\end{array}$ & 0.00 & 162.44 & 162.44 & Growth \\
\hline 2 & $\begin{array}{l}\text { Ashok } \\
\text { Leyland Ltd. }\end{array}$ & & $\begin{array}{l}\text { Automobil } \\
\text { e }\end{array}$ & $\begin{array}{l}\text { Divine } \\
\text { Heritage } \\
\text { Hotels Pvt. } \\
\text { Ltd. }\end{array}$ & $\begin{array}{l}27- \\
\text { Mar- } \\
14\end{array}$ & 3.85 & 4.54 & 17.90 & Growth \\
\hline 3 & $\begin{array}{l}\text { Power Grid } \\
\text { Corpn. Of } \\
\text { India Ltd. }\end{array}$ & \multirow{4}{*}{$\begin{array}{l}\text { Acquisition } \\
\text { of shares }\end{array}$} & Power & $\begin{array}{l}\text { Unchahar } \\
\text { Transmissio } \\
\text { n Ltd. }\end{array}$ & $\begin{array}{l}24- \\
\text { Mar- } \\
14 \\
\end{array}$ & $\begin{array}{l}49986.4 \\
6\end{array}$ & 52587.44 & 5.20 & Growth \\
\hline 4 & Oil India Ltd. & & $\begin{array}{l}\text { Petroleum } \\
\text { and } \\
\text { Natural } \\
\text { Gas. }\end{array}$ & $\begin{array}{l}\text { Indian Oil } \\
\text { Corpn. Ltd. }\end{array}$ & $\begin{array}{l}14- \\
\text { Mar- } \\
14\end{array}$ & $\begin{array}{l}28211.8 \\
8\end{array}$ & 28343.43 & 0.47 & Growth \\
\hline 5 & $\begin{array}{l}\text { R P P Infra } \\
\text { Projects Ltd. }\end{array}$ & & $\begin{array}{l}\text { Infrastruct } \\
\text { ure }\end{array}$ & $\begin{array}{l}\text { Greatful } \\
\text { Mercantile } \\
\text { Pvt. Ltd. }\end{array}$ & $\begin{array}{l}28- \\
\text { Mar- } \\
14 \\
\end{array}$ & 131.62 & 137.12 & 4.18 & Growth \\
\hline 6 & $\begin{array}{l}\text { R P P Infra } \\
\text { Projects Ltd. }\end{array}$ & & $\begin{array}{l}\text { Infrastruct } \\
\text { ure }\end{array}$ & $\begin{array}{l}\text { Birdhi } \\
\text { Chand } \\
\text { Pannalal } \\
\text { Agencies } \\
\text { Ltd. }\end{array}$ & $\begin{array}{l}28- \\
\text { Mar- } \\
14\end{array}$ & 131.62 & 137.12 & 4.18 & Growth \\
\hline
\end{tabular}




\begin{tabular}{|c|c|c|c|c|c|c|c|c|}
\hline 7 & $\begin{array}{l}\text { Sterlite Grid } \\
\text { Ltd. }\end{array}$ & $\begin{array}{l}\text { Telecom \& } \\
\text { Power Ltd. }\end{array}$ & $\begin{array}{l}\text { Rapp } \\
\text { Transmissio } \\
\text { n Co. Ltd. }\end{array}$ & $\begin{array}{l}12- \\
\text { Mar- } \\
14\end{array}$ & 793.82 & 833.26 & 4.97 & Growth \\
\hline 8 & $\begin{array}{l}\text { Surana } \\
\text { Telecom \& } \\
\text { Power Ltd. }\end{array}$ & $\begin{array}{l}\text { Telecom \& } \\
\text { Power Ltd. }\end{array}$ & $\begin{array}{l}\text { Surana } \\
\text { Solar } \\
\text { Systems } \\
\text { Pvt. Ltd. }\end{array}$ & $\begin{array}{l}21- \\
\text { Mar- } \\
14\end{array}$ & 33.33 & 32.00 & -3.99 & Decline \\
\hline 9 & U P L Ltd. & Chemical & $\begin{array}{l}\text { U P L Do } \\
\text { Brasil } \\
\text { Industria E } \\
\text { Comercio } \\
\text { De Insumos } \\
\text { Agropecuari } \\
\text { os S A }\end{array}$ & $\begin{array}{l}04- \\
\text { Mar- } \\
14\end{array}$ & $\begin{array}{l}79759.0 \\
8\end{array}$ & 79889.00 & 0.16 & Growth \\
\hline
\end{tabular}

Source: From BSE \& Annual Report with Authors' calculation

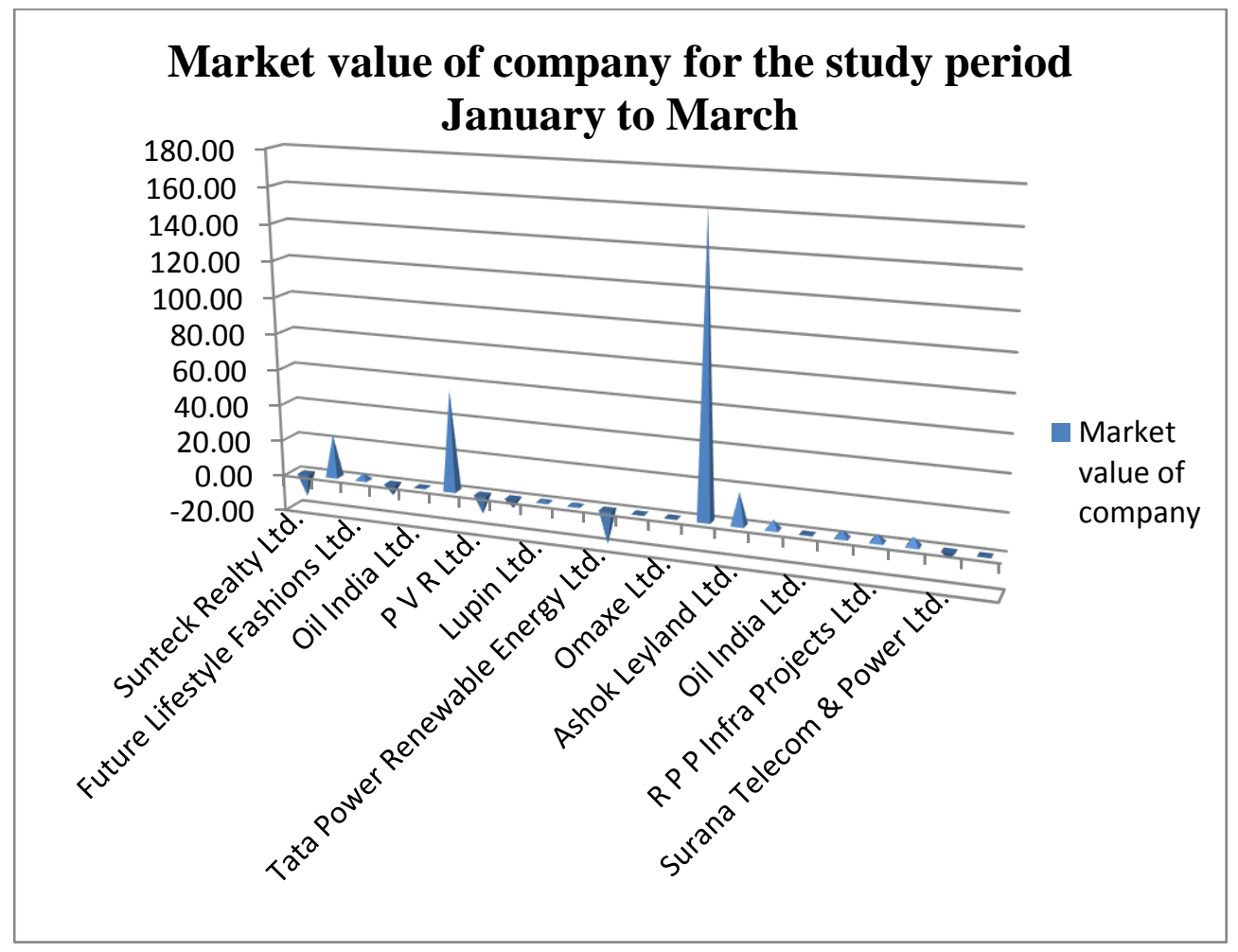




\section{Interpretation:}

The above table reveals the decline and growth of Market Value of the Company, where the average market price per share values is taken from BSE. The outstanding share values are taken from previous year annual report. In the month of January the market value of the company is increased on an average when compared with the five acquired companies, where in the month of February the market value shows a decline for majority of the selected companies. In the month of March the selected companies shows a growth as a whole.

\section{Suggestion}

The main purpose of M\&A is expanding their operation and increasing their profits. Indian markets have witnessed flourishing trend in mergers which may due to business consolidation by large industrial houses, consolidation of business by multinationals operating in India, increasing competition against imports and acquisition activities.

From this study, we can understand that many big companies are more indulged in $M \& A$, but all these $M \& A$ do not end in success there are failures too. By taking proper initiative before merger we can avoid these failures. If we focus on their creative performance of the firms, technological support, realized in lost talent, lost productivity tardiness, absenteeism, turnover, reason on their reduced output \& declining morale, loyalty, commitment and by relevant leadership they can come across these failures. Therefore, we can achieve the new entity to a long run success.

\section{Conclusion}

Mergers are considered as an important strategic tool in the global economy. The current study was based on the secondary data, where 22 merged and acquired companies have been analysed. Various attributes like Earnings per Share (EPS), Debt Equity Ratio (DER), Networth and Market value of companies were taken for this analysis, where growth percentage has been calculated.

And the findings were, the DER \& Networth shows a common growth strategy, where Market Value of Companies shows a major growth perspective but on the 
part of EPS it shows a major decline. When EPS is negative, it means the company is losing its earnings, while it typically lowers the value of stock also. Thus it can be concluded that mergers have a significant change in enhancing the 'profitability' or 'shareholders wealth' of acquirer companies.

Therefore the researcher recommends that while going for M\&A we need to check the company's each and every financial aspects and their cost values, because every single aspect is linked to various other major concepts. By checking those concepts and cost values we can determine whether we can go for a merger or acquisition deal.

\section{Note:}

\# The Annual Report values of Sterlite Technologies Limited are taken for all calculation of Sterlite Grid. As Sterlite Grid, a subsidiary of Pune based Sterlite Technologies Limited.

\# Tata Power Renewable energy is a subsidiary of Tata Power. So for calculation of MVC the BSE values are taken from Tata Power, as TPRE is not listed in BSE.

\# Ravindra Trading \& Agencies Ltd has changed its name to Ravindra Energy Ltd, before merger no trading is done in BSE.

* While calculating Average MP/S, BSE closing values were taken, for pre-merger previous month values and for post-merger, merger month is taken.

\section{Bibliography:}

1. Bhalla, P. (2011). "Determinants of Merger and Acquisitions of firms in the Indian Financial Sector: An Empirical Analysis". The IUP Journal of Business Strategy, VIII(3), 7-23.

2. Bhalla, P. (2014). "Mergers \&Acquisitions in India: A sectoral analysis" . International Journal of Business and Economic Development, 2 (2), 119-134. 
3. BharatI, P. B. (2013). "Mergers \& Acquisitions: A growth perspective from India". International Journal of Business Economics \& Management Research , 3(12), 45-58.

4. (2014). Dealtracker - providing you with M\&A and Private Equity deal insights. New Delhi: Grant thornton India.

5. Kalra, R. (2013). "Merger and Acquisitions: An Empirical Study on the Post-Merger Performance of Selected Corporate Firms in India". The IUP Journal of Business Strategy , X(4), 7-67.

6. Kumar, R. (2006). "Regulatory framework for Mergers in India: An Overview". Pranjana, $9(2)$, 93-104.

7. Kumar, R. (2009). Post-merger corporate performance: an Indian perspective. Management Research News, 32 (2), 145 - 157.

8. Mishra, P. M. (2014). "Run-up in Stock prices prior to Merger \& Acquisition Announcement: Evidence from India" . National Stock Exchange Working Paper.

9. Quan Hoang Vuong, N. K. (2014). "Relationship between Innovations, Capital Expenditures and Post -M\&A performance: Evidence from Vietnam, 2005-2012". The IUP Journal of Business Strategy , XI (1), 35-41.

10. Ramakrishnan, K. (2010). Mergers in Indian Industry: performance. Business Strategy Series, 11 (4), 261-268.

11. Raorane, P. J. (2011). "Merger and Acquisitions -A change paradigm in performance of Indian company". Sri Krishna International Research \& Educational Consortium , 2 (3), 35-51. 
12. Rathi, S. M. (2014). International Mergers \& Acquisitions Review 2014. India: Euromoney Year books-Kotak Mahindra Capital Company Limited.

13. Reddy, D. (2012). "Mergers \& Acquisitions: Initiatives to prevent failures". International Journal of Physical and Social Sciences , 2 (10), 299-309.

14. Reddy, P. M. (2008). "Type of Merger and Impact of Operating Performance: The Indian Experience" . Economic \& Political Weekly , $43(39), 66-74$.

15. Reddy, P. M. (2007). "Relative size in Mergers and Operating Performance: The Indian Experience" . Economic \& Political Weekly , 42 (39), 3936-3942.

16. Sathishkumar, R. A. (2014). "Impact of Merger and Acquisitions on Operating Performance: Evidence from Manufacturing Firms in India", Managing Global Transitions. 12 (2), 121-139.

17. Singla, A. S. (2015). "Impact of Mergers on Corporate Performance in India", No.3. Asian Journal of Research in Business Economics and Management , 5, 350-360.

18. Young, E. (2015). Transactions 2015 - Confidence spurs M\&A, 6th edition of Transaction Annual Report. Ernst \& Young LLP.

19. Zoysa, J. C. (2015). "Identify and analyse the impact of Mergers and Acquisition on operational performances and shareholder wealth - (Case study on Aviation and Banking sectors in India)". BCAS Research Symposium, 1, pp. 62-66.

20. Jens Kengelbach, Alexander Roos, and Georg Keienburg, (2014), The 2014 M\&A Report - Don't Miss the Exit 
https://www.bcgperspectives.com/content/articles/mergers_acquisi tions_divestitures_2014_m_a_report/

21. Professor Alexander, Roberts Dr William Wallace and Dr Peter Moles, "Mergers and Acquisitions", Heriot-Watt University, Edinburgh, UK, Published in Great Britain in 2010.

22. Grant Thornton India: Half Yearly 2014, 2010-2016 India Brand Equity Foundation, An initiative of the Ministry of Commerce \& Industry, Government of India, http://www.ibef.org/pages/36677

23. Matt H. Evans, Course 7: Mergers \& Acquisitions (Part 1), Excellence in Financial Management, Continuing Professional Education , http://www.exinfm.com/training/pdfiles/course07-1.pdf

24. Attorney Navpreet Panjrath, "Mergers and Acquisitions in India - A general Analysis - Corporate law http://www.legalserviceindia.com/articles/amer.htm

25. Kamal Ghosh Ray, Mergers and Acquisitions, Strategy, Valuation and Integration, 2013, PHL Learning Private Ltd, PP 1-26

\section{About the Authors}

A $R$ SINDHU, Full Time $\mathrm{Ph} \mathrm{D}$ Research Scholar, Department of Management Studies, M S University, Tirunelveli- 627 012, arsindhu25@gmail.com

DR. S. MADHAVAN, Assistant Professor, Department of Management Studies, M S University, Tirunelveli- 627 012, madhavan@msuniv.ac.in 